\title{
Detection of prostate-specific membrane antigen on HUVECs in response to breast tumor-conditioned medium
}

\author{
TIANCHENG LIU ${ }^{1}$, MOHAMED JABBES ${ }^{2}$, JESSIE R. NEDROW-BYERS ${ }^{1}$, \\ LISA Y. WU ${ }^{1}$, JEFFREY N. BRYAN ${ }^{2}$ and CLIFFORD E. BERKMAN ${ }^{1,3}$ \\ Departments of ${ }^{1}$ Chemistry and ${ }^{2}$ Veterinary Clinical Sciences, Washington State University, \\ Pullman, WA 99164; ${ }^{3}$ Cancer Targeted Technology, Woodinville, WA 98072, USA
}

Received November 25, 2010; Accepted January 24, 2011

DOI: 10.3892/ijo.2011.946

\begin{abstract}
Prostate-specific membrane antigen (PSMA), a well-known biomarker of prostate cancer, has also been found to be highly expressed in the neovasculature of multiple non-prostatic solid tumors. As a consequence, it has the potential to become a biomarker for tumor-associated vasculature. Herein, we describe an in vitro model for assessing PSMA expression associated with tube formation by primary human umbilical vein endothelial cells (HUVECs) cultured in Matrigel and induced by tumor-conditioned medium (TCM) derived from human breast cancer cells (MDA-MB-231). In contrast to vascular endothelial growth factor (VEGF)-containing endothelial cell medium, TCM induced higher expression of PSMA in HUVECs. The vessel-like tubes were detected by imaging with fluorescent PSMA inhibitors. Consequently, this in vitro model is expected to enable subsequent studies aimed at determining the role of PSMA in angiogenesis and factors that induce it.
\end{abstract}

\section{Introduction}

The cell-surface enzyme prostate-specific membrane antigen (PSMA) is an important biomarker and target in prostate cancer research (1). PSMA is up-regulated and strongly expressed on prostate tumor cells and as a consequence, it has attracted significant attention as both an imaging and therapeutic target for prostate cancer (2-4). In addition, PSMA is selectively expressed in the neovasculature of multiple non-prostatic solid tumors, including breast, kidney, bladder, lung, colon, renal, transitional cell, pancreas, neuroendocrine, and gastric cancers, but it is not expressed in normal vascular endothelium (5-8). The recent successful vascular targeting of metastatic

Correspondence to: Dr Clifford E. Berkman, Department of Chemistry, Washington State University, Pullman, WA 99164-4630, USA

E-mail: cberkman@wsu.edu

Key words: prostate-specific membrane antigen, prostate cancer, human umbilical vein endothelial cells, breast cancer tumors in patients with advanced solid tumors using the humanized anti-PSMA monoclonal antibody J591 radiolabeled with ${ }^{111}$ Indium, serves as proof-of-concept that PSMA is a valid biomarker for targeted imaging and therapy of non-prostatic tumors $(9,10)$.

The unique enzymatic activities of PSMA (11-13) and the resolution of its crystal structure (14-16) have enabled the development of various chemical inhibitor scaffolds for this enzyme-biomarker (17-24). Recently, the successful deployment of PSMA inhibitors as targeting motifs for imaging and therapeutic agents suggests that such constructs can serve as pharmacokinetic alternatives to antibodies (25-30). These studies also support the concept that such compounds may also be applied as diagnostic and therapeutic agents targeted to PSMA-positive (PSMA+) tumor-associated vasculatures of various non-prostatic tumors.

Although PSMA expression in tumor neovasculature of patients has been identified by immunohistochemical methods, there are limited examples of in vitro neovasculature models that can be used to evaluate the effectiveness of PSMA-targeted imaging or therapeutic agents. In addition, there are equally few in vitro studies that have examined the tumor factors that induce PSMA expression in vascular cells. Therefore, we set out to address these gaps in the current study. First, the tube formation capability of HUVECs (human umbilical vein endothelial cells) cultured with or without Matrigel was determined with VEGF-containing endothelial cell medium or tumor cell conditioned medium (TCM) derived from human breast cancer cells (MDA-MB-231 and MCF-7). Visualization of tube formation was obtained by phase contrast light microscopy. Second, the presence of PSMA on HUVECs cultured under the same conditions was detected by immunofluorescence analysis and further analyzed by quantitative real-time RT-PCR. We also examined the effectiveness of two fluorescent PSMA inhibitors [Fig. 1: FAM-X-CTT-54 ( $\left.\mathrm{IC}_{50}=0.35 \mathrm{nM}\right)(26)$ and Cy5.5-CTT-54 $\left(\mathrm{IC}_{50}=1.6 \mathrm{nM}\right)$ to selectively label HUVECs cultured in TCM derived from MDA-MB-231 cells]. Our data suggest that factors secreted by highly metastatic MDA-MB231 breast tumor cells potentiate PSMA expression in Matrigel-cultured HUVECs. These results are consistent with the observations that greater PSMA expression was limited to the tumor-associated neovasculature of various non-prostatic metastatic lesions. 


\section{Materials and methods}

Preparation of Cy5.5-CTT-54. The general procedure followed the protocol previously described $(26,27,31)$. In brief, a solution of $1 \mu \mathrm{mol}$ Cy5.5 mono-reactive NHS Ester (GE Healthcare Bio-Sciences Corp., Piscataway, NJ, USA) in $120 \mu \mathrm{l}$ DMSO was added to a stirred solution of the inhibitor core 1 ( $3 \mu \mathrm{mol}, 150 \mu \mathrm{l}$ of $20 \mathrm{mM}$ in $\mathrm{H}_{2} \mathrm{O}$ ), and $30 \mu \mathrm{l}$ of $1 \mathrm{M} \mathrm{NaHCO}_{3}$. The reaction mixture was stirred for $8 \mathrm{~h}$ in the dark at room temperature. The $\mathrm{pH}$ of the resulting solution was then adjusted to 9.3 by the addition of $6 \mu \mathrm{l}$ of $1 \mathrm{M} \mathrm{Na}_{2} \mathrm{CO}_{3}$. The unreacted inhibitor core 1 was scavenged by stirring with $50 \mathrm{mg}$ of SiIsocyanate resin (SiliCycle, Inc., Quebec, Canada) overnight at room temperature. The solution was subsequently centrifuged $(9,000 \mathrm{rpm}, 10 \mathrm{~min})$ and the supernatant was lyophilized in a $2 \mathrm{ml}$ microcentrifuge tube. Unreacted and/or hydrolyzed Cy5.5NHS was removed by successively triturating the lyophilized solid with $1 \mathrm{ml}$ portions of DMSO and centrifuging the mixture (1 min at 13,000 rpm) after each wash; this process was repeated 10 times. The Cy5.5-CTT-54 was dissolved in $50 \mathrm{mM}$ Tris buffer ( $\mathrm{pH}$ 7.5) to give a final concentration of $1 \mathrm{mM}$ (approximately $800 \mu \mathrm{l})$.

$I C_{50}$ determination for Cy5.5-CTT-54. Inhibition studies were performed as described previously with only minor modifications $(17,26)$. Working solutions of the substrate (N-[4-(phenylazo)-benzoyl]-glutamyl- $\gamma$-glutamic acid, PABGgG) and inhibitor were made in TRIS buffer $(50 \mathrm{mM}$, $\mathrm{pH}$ 7.4). Working solutions of purified PSMA were diluted in TRIS buffer $(50 \mathrm{mM}, \mathrm{pH} 7.4$ containing $1 \%$ Triton X-100) to provide from 15 to $20 \%$ conversion of substrate to product in the absence of inhibitor. A typical incubation mixture (final volume $250 \mu \mathrm{l}$ ) was prepared by the addition of either $25 \mu \mathrm{l}$ of an inhibitor solution or $25 \mu \mathrm{l}$ Tris buffer ( $50 \mathrm{mM}, \mathrm{pH}$ 7.4) to $175 \mu \mathrm{l}$ Tris buffer $(50 \mathrm{mM}, \mathrm{pH}$ 7.4) in a test tube. PABGgG $(25 \mu \mathrm{l}, 10 \mu \mathrm{M})$ was added to the above solution. The enzymatic reaction was initiated by the addition of $25 \mu \mathrm{l}$ of the PSMA working solution. In all cases, the final concentration of PABGgG was $1 \mu \mathrm{M}$ while the enzyme was incubated with five serially diluted inhibitor concentrations providing a range of inhibition from 10 to $90 \%$. The reaction was allowed to proceed for $15 \mathrm{~min}$ with constant shaking at $37^{\circ} \mathrm{C}$ and was terminated by the addition of $25 \mu \mathrm{l}$ methanolic TFA ( $2 \%$ trifluoroacetic acid by volume in methanol) followed by vortexing. The quenched incubation mixture was quickly buffered by the addition of $25 \mu \mathrm{l}$ $\mathrm{K}_{2} \mathrm{HPO}_{4}(0.1 \mathrm{M})$, vortexed, and centrifuged (10 min at 7,000 g). An $85 \mu \mathrm{l}$ aliquot of the resulting supernatant was subsequently quantified by HPLC as previously described $(32,33)$. $\mathrm{IC}_{50}$ values were calculated using Kaleida Graph 3.6 (Synergy Software).

Endothelial cell culture. Primary human umbilical vein endothelial cells (HUVECs) were obtained from Lifeline Cell Technology (Walkersville, MD), and cultured in complete VascuLife medium (Lifeline Cell Technology) containing 2\% fetal bovine serum (FBS), $10 \mathrm{mM}$ L-glutamine, $5 \mathrm{ng} / \mathrm{ml} \mathrm{recom-}$ binant human vascular endothelial growth factor (rh-VEGF), $5 \mathrm{ng} / \mathrm{ml}$ recombinant human epidermal growth factor (rh-EGF), $5 \mathrm{ng} / \mathrm{ml}$ recombinant human basic fibroblast growth factor (rh-bFGF), $15 \mathrm{ng} / \mathrm{ml}$ recombinant human insulin-like growth factor 1 (IGF-1), $50 \mu \mathrm{g} / \mathrm{ml}$ ascorbic acid, $1 \mu \mathrm{g} / \mathrm{ml}$ hydrocortisone hemisuccinate, and 0.75 units $/ \mathrm{ml}$ heparin sulfate, and maintained in a humidified incubator at $37^{\circ} \mathrm{C}$ and $5 \% \mathrm{CO}_{2}$. HUVECs may be sub-cultured by detachment of $0.05 \%$ Trypsin- $0.02 \%$ EDTA solution once the culture is $80-90 \%$ confluent and actively proliferating. HUVECs passaged 3-6 times were successfully archived with the freezing medium (10\% DMSO, 10\% FBS in VascuLife medium) for future use.

Preparation of tumor cell conditioned medium. This protocol was based on a previous report with minor modifications (34). The breast cancer cell lines MCF-7 and MDA-MB-231 were respective gifts from Dr Margaret E. Black and Dr David W. Koh (Washington State University-Pullman). The human prostate cancer cell lines LNCaP and PC-3 were obtained from the American Type Culture Collection (Manassas, VA). The breast cell lines were cultured in Eagle's minimum Essential medium (EMEM) with 10\% FBS in T75 flasks in a humidified incubator at $37^{\circ} \mathrm{C}$ and $5 \% \mathrm{CO}_{2}$. The prostate cancer cell lines were cultured under the same conditions except in RPMI-1640 medium. At $100 \%$ confluence of cell monolayer, the old medium was removed, and cells were washed three times in serum-free RPMI-1640 and incubated with $10 \mathrm{ml}$ serum-free RPMI-1640 each flask for $24 \mathrm{~h}$. Tumor cell conditioned medium (TCM) was centrifuged for $10 \mathrm{~min}$ at $3,000 \mathrm{~g}\left(4^{\circ} \mathrm{C}\right)$ and the supernatants were collected, filtered through a $0.22 \mu \mathrm{m}$ filter, and stored at $-20^{\circ} \mathrm{C}$ until use.

Tube formation on Matrigel. The day before the fluorescencelabeling experiments, growth factor-reduced Matrigel (BD Biosciences, Bedford, MA) was thawed overnight at $4^{\circ} \mathrm{C}$ on ice and, the day of experiment, plated on the bottom of $\mathrm{BD}$ Falcon 2-well Cultureslides at $200 \mu \mathrm{l} / \mathrm{each}$ well, and left at $37^{\circ} \mathrm{C}$ for $30 \mathrm{~min}$ for gelification. Approximately $10^{5}$ cells/well were seeded on Matrigel-coated 2-well Cultureslides and incubated for $18 \mathrm{~h}$ with $2 \mathrm{ml}$ complete VascuLife medium or TCM in a humidified incubator at $37^{\circ} \mathrm{C}$ and $5 \% \mathrm{CO}_{2}$. The tube formation was visualized using a compound light microscope (Olympus BH-2, Olympus Optical Co. Ltd., Tokyo, Japan) at X20 magnification (objective lens numerical aperture $=1.25$ ). Digital images were obtained using a digital camera system (Jenoptik ProgRes Camera, C12plus, JENOPTIK Laser, Optik, System GmbH, Jena, Germany) mounted on the micro-scope.

Immunocytochemistry detection of PSMA. The mouse monoclonal antibody 7E11 was provided by Cytogen Corporation (Princeton, NJ). The goat anti-mouse secondary antibodyTRIC was obtained from Jackson ImmunoResearch Laboratories (West Grove, PA). Immunofluorescence detection of PSMA was conducted as we previously described (24). As a positive control for this method of detection, LNCaP cells were cultured for 3 days on the slides under normal conditions (24). HUVECs cultured on the slides with or without Matrigel were washed twice in 1X PBS buffer (phosphate buffered saline), fixed in 4\% paraformaldehyde in PBS for $20 \mathrm{~min}$ at room temperature, and permeabilized with cold-methanol for $5 \mathrm{~min}$ at $-20^{\circ} \mathrm{C}$. The fixed cells were blocked with $1 \%$ BSA, PBS for $30 \mathrm{~min}$ and incubated with primary antibody 7E11 (300X diluted in PBS containing $1 \% \mathrm{BSA}$ ) for $60 \mathrm{~min}$ at room temperature. After washing, the cells were incubated with a secondary antibody 

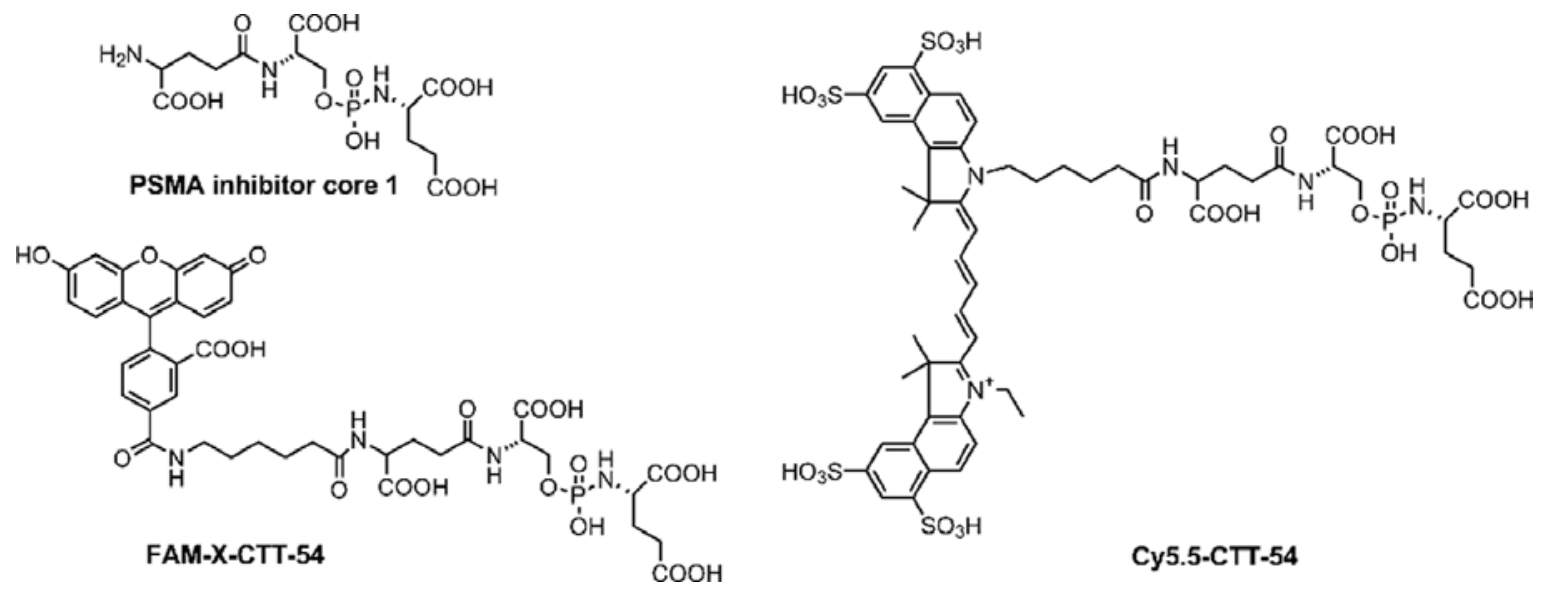

Figure 1. Structures of phosphoramidate peptidomimetic inhibitor core 1, and its fluorescent dye conjugates: FAM-X-CTT-54 and Cy5.5-CTT-54.

(goat anti-mouse IgG-TRITC, 50X diluted in 1\% BSA, PBS) for $60 \mathrm{~min}$, counterstained with Hoechst 33342 (InvitrogenMolecular Probes, Carlsbad, CA), and mounted in Vectashield ${ }^{\circledR}$ Mounting Medium (Vector Laboratories, Inc., Burlingame, CA) for confocal microscopy.

Cell-labeling with fluorescent PSMA inhibitors. Cells cultured on the 2-well Culture slides with or without Matrigel were washed twice with warm medium A (phosphate-free RPMI1640 containing $1 \% \mathrm{FBS}$ ), then incubated with $1 \mathrm{ml}$ of either fluorescent inhibitor $(10 \mu \mathrm{M})$ in warm medium $\mathrm{A}$ for $2 \mathrm{~h}$ in a humidified incubator at $37^{\circ} \mathrm{C}$ and $5 \% \mathrm{CO}_{2}$. All the above treated cells were washed three times with cold-KRB buffer $\mathrm{pH} 7.4$ (mmol/l: $\mathrm{NaCl}$ 154.0, $\mathrm{KCl}$ 5.0, $\mathrm{CaCl}_{2}$ 2.0, $\mathrm{MgCl}_{2}$ 1.0, HEPES 5.0, D-glucose 5.0) and fixed with $4 \%$ paraformaldehyde in KRB for $20 \mathrm{~min}$ at room temperature. The cellular nuclei were counterstained with Hoechst 33342, then the cells were mounted in Vectashield Mounting Medium for microscopy.

Fraction of tumor cell conditioned medium. The MDA-MB$231 \mathrm{TCM}$ was fractioned through 30, 10 and $3 \mathrm{kDa}$ molecular weight cut-off centrifugal filter devices (Millipore Corporation, Bedford, MA) according to the manufacturer's instructions. The fractions: $>30,10-30,3-10$ and $<3 \mathrm{kDa}$ were collected and stored at $-20^{\circ} \mathrm{C}$ until use. The effects on tube formation and PSMA expression of HUVECs in the presence of the individual fractions with Matrigel were evaluated.

Confocal laser scanning microscopy. Cells were visualized under 25x (for FAM-X-CTT-54) or 40x (for Cy5.5-CTT-54) oil immersion objective using an LSM 510 META laser Scanning Microscope. Hoechst 33342 was excited with a Diode laser (405 nm) and the emission collected with a BP420-480 nm filter. FAM-X-CTT-54 staining was excited using $488 \mathrm{~nm}$ from an Argon laser, and the emission collected with a LP505 nm filter. PSMA immunofluorescence was excited using 543 $\mathrm{nm}$ from a HeNe laser, and the emission collected with a BP560-615 nm filter. Cy5.5-CTT-54 staining was excited using $633 \mathrm{~nm}$ from a HeNe laser, and the emission collected with a LP $650 \mathrm{~nm}$ filter. To reduce interchannel crosstalk, a multi-tracking technique was used and images were taken at a resolution of $1,024 \times 1,024$ pixels. Confocal scanning parameters were set up so that the control cells without treatment had no fluorescent signal from background. The imaging colors of fluorescent Dyes (Hoechst 33342, FAM and TRITC) were defined as blue, green and red, respectively. As the emission wavelength of the near-infrared fluorescent dye Cy5.5 is beyond visible ranges, fluorescence pseudocolor of Cy5.5 was assigned as red. The pictures were edited by National Institutes of Health (NIH) Image J software (http://rsb.info.nih. gov/ij) and Adobe Photoshop CS2.

Quantitative real-time RT-PCR. HUVECs cultured in Matrigel were released without damage and recovered using BD MatriSperse Cell recovery solution (BD Biosciences, Franklin Lakes, NJ). Total RNA was extracted using the RNeasy kit (Qiagen, Valencia, CA) from HUVECs cultured under experimental conditions in MCF-7 or MDA-MB-231 cell TCM, or VascuLife media with VEGF added and from LNCaP cells as a positive control. Contaminating DNA was removed using TURBO DNA free kit (Ambion, Austin, TX). RNA was cleaned to yield high purity. RNA was converted to cDNA using the SuperScript Vilo cDNA synthesis kit (Invitrogen, Carlsbad, CA). The PSMA primers and TaqMan MGB probes were designed with primer Express 3.0 (Applied Biosystems, USA). In addition the PSMA primers were also designed to span first intron region to avoid DNA contamination and differentiate PSMA from PSM' (35). cDNA was amplified by PCR using the primers PSMA151F (5'-CCCTTCATTGACCTCAACTACATGGT-3') and PSMA472 R (5'-TGATGTTCTCAGCTTTCAA TTCATCC-3'). The product was cloned into competent vectors using the Topo-TA cloning kit (Invitrogen, Carlsbad, CA). The sequence of the insert in the clones was confirmed with dideoxynucleotide sequencing. Quantitative real-time PCR was performed as previously described (36). A standard curve for the PSMA gene was constructed with five 10 -fold serial dilutions of the plasmid from $3.0 \times 10^{7}$ to $3.0 \times 10^{3}$. The experimental samples were amplified with following primer pair PSMA343F (5'-TGGCGGGTGGCTTCTTT-3') and PSMA423R (5'-AGG AGTAATGTTAGTAGCTTCATTGGAGG-3'), and the PCR products were further quantified with the probe PSMA173P containing a fluorophore and quencher (5' FAM-CTCTTCGG 


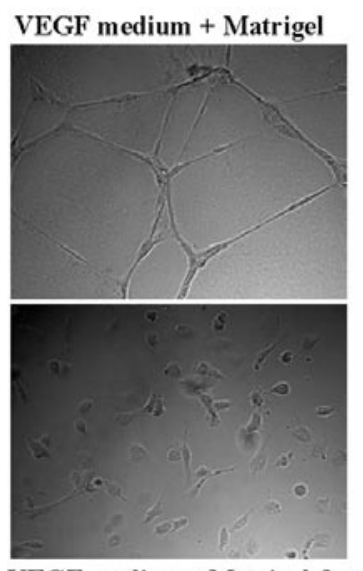

VEGF medium + Matrigel-free

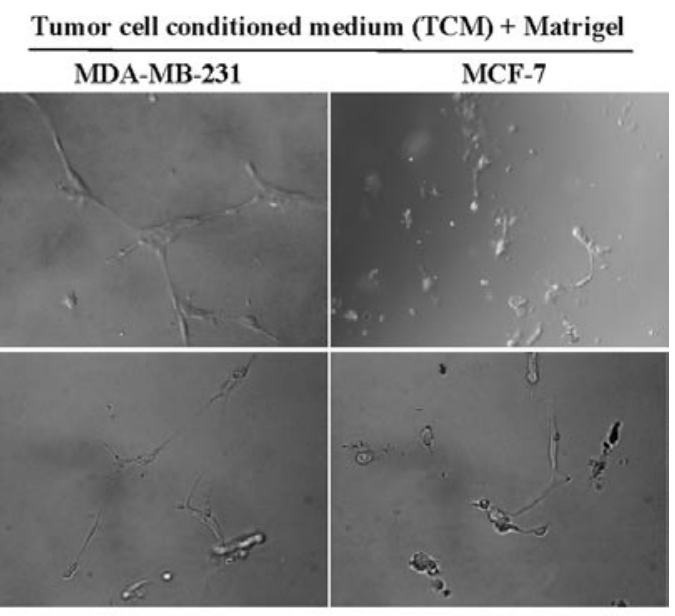

PC-3
LNCaP

Figure 2. Tube formation by HUVECs on Matrigel. HUVECs were plated on Matrigel in the presence of VEGF-containing VascuLife medium, tumor cell conditioned medium (from MDA-MB-231, MCF-7, PC-3 and LNCaP, respectively). HUVECs cultured in the presence of VEGF-containing VascuLife medium but in the absence of Matrigel served as control samples. The phase contrast photographs were captured by an inverted light microscope at magnification $\mathrm{x} 20$.

A

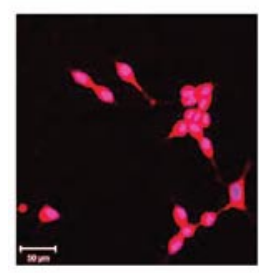

B

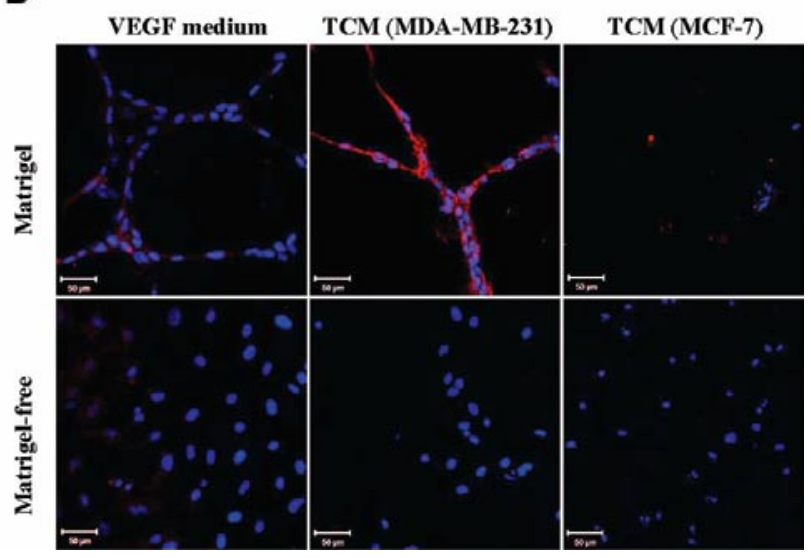

Figure 3. Immunofluorescence detection of PSMA on HUVECs. (A) LNCaP cells as a PSMA-positive control sample. (B) HUVECs plated with or without Matrigel in the presence of VEGF-containing VascuLife medium or tumor cell conditioned medium from MDA-MB-231 and MCF-7 cells. Immunofluorescence signals (red) for PSMA were clearly detected on the tube-like formations by HUVECs cultured in TCM from MDA-MB-231 cells with less PSMA detected on HUVECs cultured in either Matrigel in VEGF-contained VascuLife medium or TCM from MCF-7 cells. No PSMA was detected on HUVECs cultured in the absence of Matrigel. Cell nuclei were counterstained with Hoechst 33342 (blue). The cellular imaging was visualized by confocal microscopy; distance scale is $50 \mu \mathrm{m}$.

GTGGTTTAT-MGB 3'). A standard threshold was set and CT values were converted to number of copies per $\mu \mathrm{g}$ of RNA. Differences were tested for significance using a one-way ANOVA with significance set at $\mathrm{P}<0.05$.

\section{Results}

TCM-induced tube formation. HUVECs seeded on Matrigel formed vessel-like tube formation networks in VEGF-containing VascuLife medium. The tube formation also was observed for HUVECs cultured in TCM from MDA-MB-231 cells. In contrast, HUVECs formed only incomplete, short tube-like structures in TCM from MCF-7, PC-3, and LNCaP cells, or cultured separately without Matrigel in VEGF-containing VascuLife medium (Fig. 2).
PSMA expression in TCM-induced vessel-like tubes. The mouse monoclonal anti-PSMA antibody 7E11, specifically binds to the cytoplasmic domain of PSMA and has been widely utilized to detect PSMA by Western blotting or by immunofluorescence $(11,37)$. In a positive control study, a strong fluorescence signal for PSMA on LNCaP cells was detected using 7E11-based immunofluorescence (Fig. 3A). This same protocol was further employed to detect PSMA on HUVECs. Compared with little or no detectable PSMA on HUVECs cultured in VEGFcontaining VascuLife medium or MCF-7 TCM, with or without Matrigel, considerable expression of PSMA in MDA-MB-231 TCM-induced vessel-like tubes was detected by immunofluorescence. On the other hand, only MDA-MB-231 TCM without Matrigel cannot induce PSMA expression on HUVECs (Fig. 3B). It also was noted that HUVECs were not viable in 
A

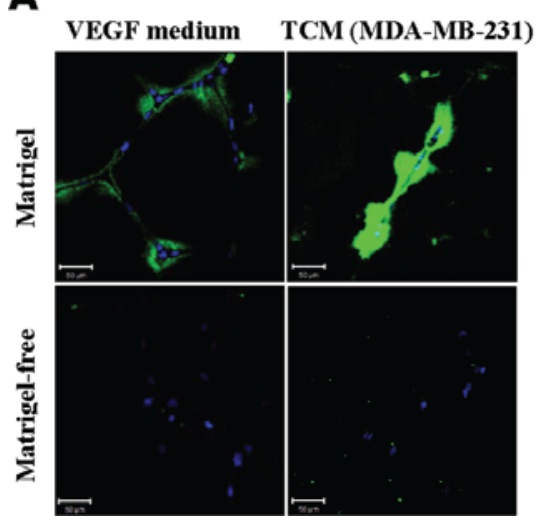

B

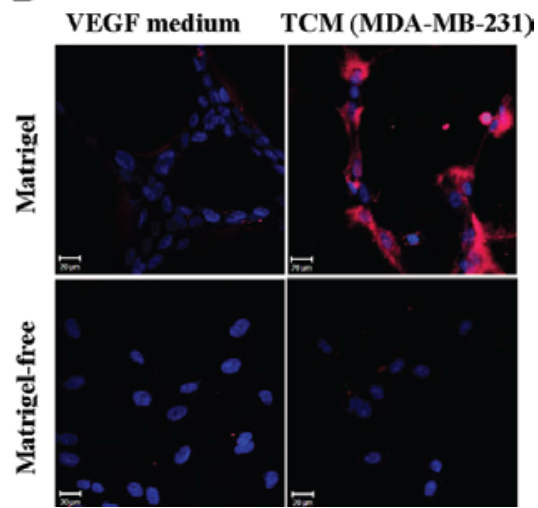

Figure 4. Fluorescent inhibitor-affinity labeling of PSMA in HUVECs. HUVECs were plated with or without Matrigel in the presence of VEGF-contained VascuLife medium or tumor conditioned medium from MDA-MB-231 cells. Both FAM-X-CTT-54 labeling (green, A) and Cy5.5-CTT-54 labeling (red, B) of PSMA was clearly detected in TCM-induced tubes with less on HUVECs cultured in Matrigel in the presence of VEGF-contained VascuLife medium, and none observed on HUVECs cultured in Matrigel-free conditions. Cell nuclei were counterstained with Hoechst 33342 (blue). Cellular imaging of FAM-X-CTT-54 labeling was visualized at magnification x 25 by confocal microscopy, distance scale is $50 \mu \mathrm{m}$. Cellular imaging of Cy5.5-CTT-54 labeling was visualized at magnification $\mathrm{x} 40$ by confocal microscopy, distance scale is $20 \mu \mathrm{m}$.

The fractions of tumor cell conditioned medium from MDA-MB-231

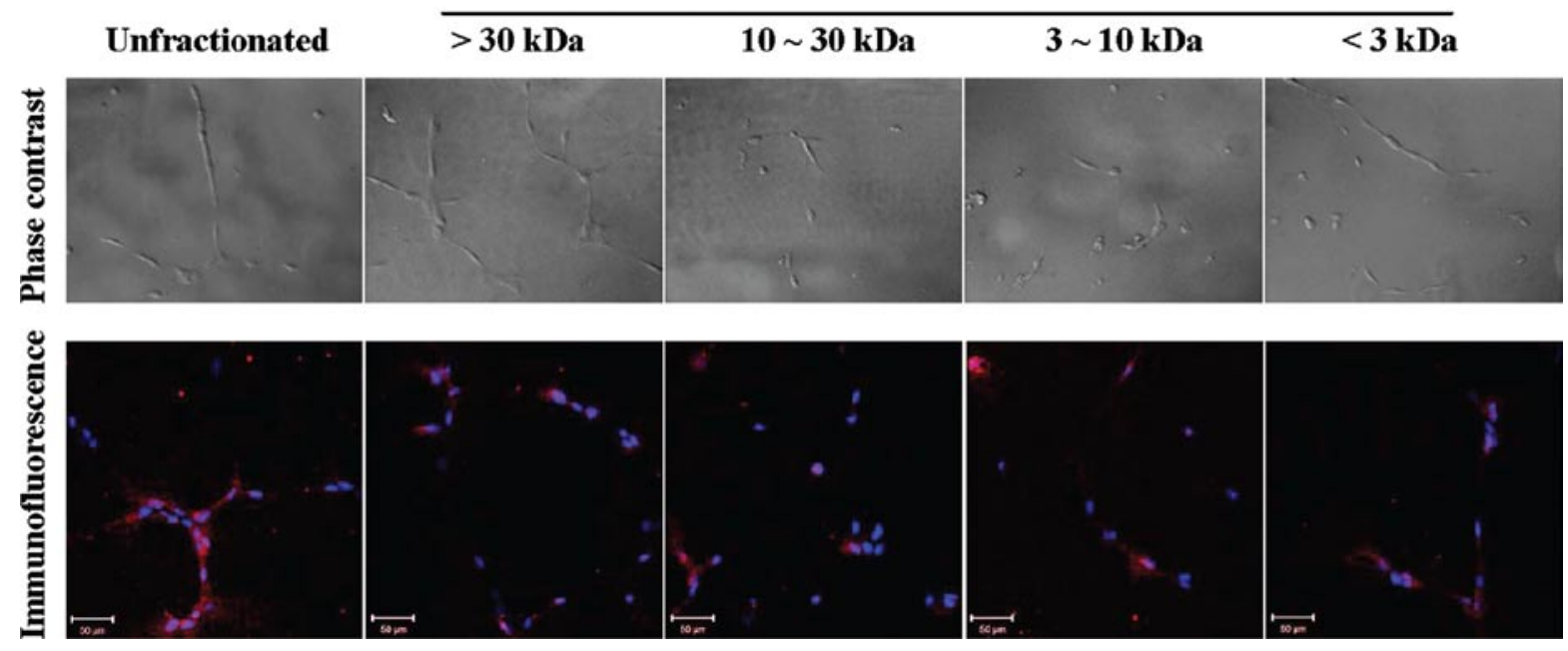

Figure 5. The effects on tube formation and PSMA expression with fractions of tumor conditioned medium from MDA-MB-231 cells. HUVECs were plated on Matrigel in the presence of TCM from MDA-MB-231 (unfractionated) and decreasing size fractions. (Top) Phase contrast photographs were captured by an inverted light microscope at magnification x20. (Bottom) Cellular immunofluorescence of PSMA was visualized at magnification x25 by confocal microscopy. The nuclei were counterstained with Hoechst 33342 (blue). The distance scale is $50 \mu \mathrm{m}$.

MCF-7 TCM without Matrigel, which were characterized by nuclear shrinking and fragmentation (Fig. 3B). Based on the above results, it is clear that presently unknown factors secreted by MDA-MB-231 induces both tube formation and PSMA expression for HUVECs cultured on Matrigel, and also support the viability of HUVECs cultured in the absence of Matrigel.

Affinity labeling of PSMA in TCM-induced vessel-like tubes. FAM-X-CTT-54, a specific PSMA fluorescent inhibitor was designed and evaluated for PSMA-targeted fluorescence imaging of LNCaP cells in our previous studies $(24,26)$. In this study, a new, near infrared fluorescence inhibitor Cy5.5CTT-54 was synthesized and its high inhibition against PSMA was revealed with $\mathrm{IC}_{50}$ value at $1.6 \mathrm{nM}$. Furthermore, FAM-XCTT-54 and Cy5.5-CTT-54 were employed for fluorescence imaging of PSMA-positive HUVECs. Consistent with the results for the immunofluorescence study above, the greatest labeling by these fluorescent PSMA inhibitors was observed for MDA-MB-231 TCM-induced vessel-like tubes with Matrigel, weak signals for HUVECs cultured on Matrigel in VEGFcontaining VascuLife medium, and no signal detected for matrigel-free VEGF-containing VascuLife medium or MDA-MB-231 TCM cultured HUVECs (Fig. 4).

The cellular effects of fractioned TCM. The MDA-MB-231 TCM-induced vessel-like tube formation was dependent on factors from two fractions from the TCM: $>30$ and more 
interestingly, $<3 \mathrm{kDa}$ (Fig. 5). Furthermore, the effect of two fractions is additive in inducing PSMA expression (Fig. 5).

Quantitative real-time RT-PCR. The experimental and standard curve samples amplified with $92.1 \%$ efficiency. HUVECs cultured in MDA-MB-231 TCM expressed $3.72 \times 10^{5}$ (SEM $\left.1.42 \times 10^{4}\right)$ copies of PSMA per $\mu \mathrm{g}$ of RNA. HUVECs cultured in VEGF-containing VascuLife medium expressed 2.11 $\times 10^{5}$ (SEM $1.80 \times 10^{4}$ ) copies of PSMA per $\mu \mathrm{g}$ of RNA. HUVECs

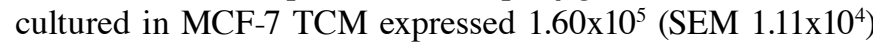
copies of PSMA per $\mu \mathrm{g}$ of RNA. LNCaP cells expressed $8.31 \times 10^{7}$ (SEM $2.53 \times 10^{6}$ ) copies of PSMA per $\mu \mathrm{g}$ of RNA. Differences between groups were significant $(\mathrm{P}<0.001)$.

\section{Discussion}

Estrogen receptor negative (ER-) breast cancer has been clinically recognized as being more aggressive and having a poorer prognosis than $\mathrm{ER}^{+}$breast cancer. In our current study, MDA-MB-231 (ER') and MCF-7 (ER+) cells were chosen as in vitro models to represent these two different progressive stages of breast cancer. Based on our data, MDA-MB-231 (ER-) cells promoted greater angiogenesis with higher PSMA mRNA transcription and protein expression in HUVECs cultured in Matrigel than MCF-7 (ER $)$ cells. These results are consistent with the higher levels of VEGF and MMP-9 secreted by MDAMB-231 cells compared to MCF-7 cells $(34,38)$.

With respect to the enzyme-biomarker PSMA, an interesting question now remains as to what in TCM from MDA-MB-231 is correlated with greater expression of PSMA in HUVECs. Based on the present PSMA immunfluorescence and affinitylabeling data for HUVECs cultured in Matrigel with VEGF-containing medium, it is apparent that VEGF levels are not mainly correlated with PSMA expression as only weak signals were observed for these conditions. Interestingly, TCM from MDA-MB-231 cells was unable to induce any detectable level of PSMA on HUVECs cultured in the absence of Matrigel. Therefore, our current data suggest that factors both $>30$ and $<3 \mathrm{kDa}$ from TCM secreted by MDA-MB-231 cells in combination with the Matrigel environment induces PSMA expression on HUVECs.

Inducible PSMA specific expression on angiogenic vasculature suggests that PSMA participates in neovessel growth in developing tumors. Experimental evidence to further support this hypothesis is based on results from Shapiro's group in which they observed that in either PSMA-null mice, or in PSMA wild-type mice treated with a PSMA inhibitor, angiogenesis was significantly impaired in the Matrigel implants (39). In our current study, in vitro PSMA expression on cultured HUVECs was specifically limited to growth conditions with MDA-MB-231-TCM in Matrigel. Together these data suggest that PSMA may also be a clinically relevant biomarker for tumors undergoing vascularization and consequently a predictive marker for those tumors likely to respond to antiangiogenic therapy. The successful in vitro model described herein is expected to enable further studies that elucidate the relationship between PSMA expression and tumor angiogenesis with a goal to develop tumor-vascular targeting agents for imaging and therapeutic applications.

\section{Acknowledgements}

We thank Cytogen Corporation (Princeton, NJ) for the gift of the mouse monoclonal antibody 7E11, and extend our gratitude for technical assistance to G. Helms and W. Hiscox at the WSU Center for NMR Spectroscopy, as well as C. Davitt and V. Lynch-Holm at the WSU Franceschi Microscopy and Imaging Center.

\section{References}

1. Ghosh A and Heston WD: Tumor target prostate specific membrane antigen (PSMA) and its regulation in prostate cancer. J Cell Biochem 91: 528-539, 2004.

2. Tagawa ST, Beltran H, Vallabhajosula S, et al: Anti-prostatespecific membrane antigen-based radioimmunotherapy for prostate cancer. Cancer 116: 1075-1083, 2010.

3. Bander NH: Technology insight: monoclonal antibody imaging of prostate cancer. Nat Clin Pract Urol 3: 216-225, 2006.

4. Nanus DM, Milowsky MI, Kostakoglu L, et al: Clinical use of monoclonal antibody HuJ591 therapy: targeting prostate specific membrane antigen. J Urol 170: S84-S89, 2003.

5. Chang SS, O'Keefe DS, Bacich DJ, Reuter VE, Heston WD and Gaudin PB: Prostate-specific membrane antigen is produced in tumor-associated neovasculature. Clin Cancer Res 5: 2674-2681, 1999.

6. Haffner MC, Kronberger IE, Ross JS, et al: Prostate-specific membrane antigen expression in the neovasculature of gastric and colorectal cancers. Hum Pathol 40: 1754-1761, 2009.

7. Chang SS, Reuter VE, Heston WD, Bander NH, Grauer LS and Gaudin PB: Five different anti-prostate-specific membrane antigen (PSMA) antibodies confirm PSMA expression in tumorassociated neovasculature. Cancer Res 59: 3192-3198, 1999.

8. Liu H, Moy P, Kim S, et al: Monoclonal antibodies to the extracellular domain of prostate-specific membrane antigen also react with tumor vascular endothelium. Cancer Res 57: 3629-3634, 1997.

9. Milowsky MI, Nanus DM, Kostakoglu L, et al: Vascular targeted therapy with anti-prostate-specific membrane antigen monoclonal antibody J591 in advanced solid tumors. J Clin Oncol 25: 540-547, 2007.

10. Morris MJ, Pandit-Taskar N, Divgi CR, et al: Phase I evaluation of J591 as a vascular targeting agent in progressive solid tumors. Clin Cancer Res 13: 2707-2713, 2007.

11. Grauer LS, Lawler KD, Marignac JL, Kumar A, Goel AS and Wolfert RL: Identification, purification, and subcellular localization of prostate-specific membrane antigen PSM' protein in the LNCaP prostatic carcinoma cell line. Cancer Res 58: 4787-4789, 1998.

12. Carter RE, Feldman AR and Coyle JT: Prostate-specific membrane antigen is a hydrolase with substrate and pharmacologic characteristics of a neuropeptidase. Proc Natl Acad Sci USA 93: 749-753, 1996.

13. Pinto JT, Suffoletto BP, Berzin TM, et al: Prostate-specific membrane antigen: a novel folate hydrolase in human prostatic carcinoma cells. Clin Cancer Res 2: 1445-1451, 1996.

14. Mesters JR, Henning K and Hilgenfeld R: Human glutamate carboxypeptidase II inhibition: structures of GCPII in complex with two potent inhibitors, quisqualate and 2-PMPA. Acta Crystallogr D Biol Crystallogr 63: 508-513, 2007.

15. Davis MI, Bennett MJ, Thomas LM and Bjorkman PJ: Crystal structure of prostate-specific membrane antigen, a tumor marker and peptidase. Proc Natl Acad Sci USA 102: 5981-5986, 2005.

16. Mesters JR, Barinka C, Li W, et al: Structure of glutamate carboxypeptidase II, a drug target in neuronal damage and prostate cancer. EMBO J 25: 1375-1384, 2006.

17. Wu LY, Anderson MO, Toriyabe Y, et al: The molecular pruning of a phosphoramidate peptidomimetic inhibitor of prostatespecific membrane antigen. Bioorg Med Chem 15: 7434-7443, 2007.

18. Ding P, Helquist $P$ and Miller MJ: Design, synthesis and pharmacological activity of novel enantiomerically pure phosphonic acid-based NAALADase inhibitors. Org Biomol Chem 5: 826-831, 2007. 
19. Majer P, Hin B, Stoermer D, et al: Structural optimization of thiol-based inhibitors of glutamate carboxypeptidase II by modification of the P1' side chain. J Med Chem 49: 2876-2885, 2006.

20. Aggarwal S, Singh P, Topaloglu O, Isaacs JT and Denmeade SR A dimeric peptide that binds selectively to prostate-specific membrane antigen and inhibits its enzymatic activity. Cancer Res 66: 9171-9177, 2006.

21. Wone DW, Rowley JA, Garofalo AW and Berkman CE: Optimizing phenylethylphosphonamidates for the inhibition of prostate-specific membrane antigen. Bioorg Med Chem 14: 67-76, 2006

22. Zhou J, Neale JH, Pomper MG and Kozikowski AP: NAAG peptidase inhibitors and their potential for diagnosis and therapy. Nat Rev Drug Discov 4: 1015-1026, 2005.

23. Tsukamoto T, Wozniak KM and Slusher BS: Progress in the discovery and development of glutamate carboxypeptidase II inhibitors. Drug Discov Today 12: 767-776, 2007.

24. Liu T, Toriyabe Y, Kazak M and Berkman CE: Pseudoirreversible inhibition of prostate-specific membrane antigen by phosphoramidate peptidomimetics. Biochemistry 47: 12658-12660, 2008.

25. Liu T, Wu LY, Hopkins MR, Choi JK and Berkman CE: A targeted low molecular weight near-infrared fluorescent probe for prostate cancer. Bioorg Med Chem Lett 20: 7124-7126, 2010.

26. Liu T, Wu LY, Kazak M and Berkman CE: Cell-surface labeling and internalization by a fluorescent inhibitor of prostate-specific membrane antigen. Prostate 68: 955-964, 2008.

27. Liu T, Wu LY, Choi JK and Berkman CE: In vitro targeted photodynamic therapy with a pyropheophorbide - a conjugated inhibitor of prostate-specific membrane antigen. Prostate 69: 585-594, 2009

28. Lapi SE, Wahnishe H, Pham D, et al: Assessment of an 18F-labeled phosphoramidate peptidomimetic as a new prostatespecific membrane antigen-targeted imaging agent for prostate cancer. J Nucl Med 50: 2042-2048, 2009.

29. Hillier SM, Maresca KP, Femia FJ, et al: Preclinical evaluation of novel glutamate-urea-lysine analogues that target prostatespecific membrane antigen as molecular imaging pharmaceuticals for prostate cancer. Cancer Res 69: 6932-6940,2009.
30. Kularatne SA, Wang K, Santhapuram HK and Low PS: Prostatespecific membrane antigen targeted imaging and therapy of prostate cancer using a PSMA inhibitor as a homing ligand. Mol Pharm 6: 780-789, 2009.

31. Liu T, Wu LY, Choi JK and Berkman CE: Targeted photodynamic therapy for prostate cancer: Inducing apoptosis via activation of the caspase-8/-3 cascade pathway. Int J Oncol 36: 777-784, 2010.

32. Anderson MO, Wu LY, Santiago NM, et al: Substrate specificity of prostate-specific membrane antigen. Bioorg Med Chem 15: 6678-6686, 2007.

33. Maung J, Mallari JP, Girtsman TA, et al: Probing for a hydrophobic a binding register in prostate-specific membrane antigen with phenylalkylphosphonamidates. Bioorg Med Chem 12: 4969-4979, 2004.

34. Marchetti M, Vignoli A, Russo L, et al: Endothelial capillary tube formation and cell proliferation induced by tumor cells are affected by low molecular weight heparins and unfractionated heparin. Thromb Res 121: 637-645, 2008.

35. Zhang L, Wang CY, Yang R, et al: Real-time quantitative RT-PCR assay of prostate-specific antigen and prostate-specific membrane antigen in peripheral blood for detection of prostate cancer micrometastasis. Urol Oncol 26: 634-640, 2008.

36. Chang X, Han J, Pang L, Zhao Y, Yang Y and Shen Z: Increased PADI4 expression in blood and tissues of patients with malignant tumors. BMC Cancer 9: 40, 2009.

37. Troyer JK, Beckett ML and Wright GL Jr: Location of prostatespecific membrane antigen in the LNCaP prostate carcinoma cell line. Prostate 30: 232-242, 1997.

38. Wang XY, Tan JX, Vasse M, Delpech B and Ren GS: Comparison of hyaluronidase expression, invasiveness and tubule formation promotion in ER (-) and ER (+) breast cancer cell lines in vitro. Chin Med J (Engl) 122: 1300-1304, 2009.

39. Conway RE, Petrovic N, Li Z, Heston W, Wu D and Shapiro LH: Prostate-specific membrane antigen regulates angiogenesis by modulating integrin signal transduction. Mol Cell Biol 26: 5310-5324, 2006 